\title{
EXPLORING THE LIMITS OF PHASE REFERENCED VLBI IMAGING; A PROGRESS REPORT
}

\author{
R.C. VERMEULEN ${ }^{1}$, J.E. CONWAY ${ }^{1,2}$ \\ ${ }^{1}$ Owens Valley Radio Observatory, ${ }^{2}$ Jet Propulsion Laboratory, \\ California Institute of Technology, Pasadena, CA 91125, U.S.A.
}

\begin{abstract}
The practical limits of phase referenced VLBI imaging are not well known. If it would be feasible to use target-reference separations of a few degrees, most of the sky would be accessible to the technique. Preliminary results at $6 \mathrm{~cm}$ using baselines of up to $1000 \mathrm{~km}$ are encouraging.
\end{abstract}

\section{GOALS OF THE PROJECT}

We are exploring the feasibility of using phase-referencing to increase the coherence time of a VLBI network from minutes to hours, so as to be able to detect, and then image, very weak $(\sim \mathrm{mJy})$ sources. Catalogues of phase calibrators, with accurate positions, are now being compiled (e.g., Patnaik et al., these proceedings). On average, a suitably strong $(\sim 200 \mathrm{mJy})$ compact source can be found within $\sim 3^{\circ}$ from any desired target. We have designed experiments to explore whether such separations can be used, how bright the reference source should be, and how rapidly switching must take place (compare Alef 1988). We also hope to investigate how the accuracy of phase calibration depends on baseline length, weather conditions, and time of day.

\section{THE EXPERIMENTS}

We use 3 C 345 as a phase reference for $1638+398,1652+398,1633+382$, and $1624+416$, which are separated from it by $0.4^{\circ}, 1.5^{\circ}, 2.2^{\circ}$, and $3.7^{\circ}$ respectively. The position of all sources is known to a few mas. They all have a total flux density $>1 \mathrm{Jy}$, and so can be detected with high signal-to-noise in 1-minute scans using Mark II recording. Using these sources we are able to compare the results of conventional fringe-fitting and hybrid mapping to phase referenced mapping, and to assess the effects of source structure phase. To probe temporal variability on the smallest accessible scales, we use a cycle time of 3 minutes; effectively, we obtain $\sim 1$ minute of data on each source. We observe the source pairs in sections of $\sim 15$ contiguous cycles ( $\sim 45$ minutes), thus obtaining data on each target roughly every 3 hours (i.e. several times during a day).

We report here on two 6 - $\mathrm{cm}$ observations. In November 1989 we obtained 4 hours of data (1 section per source pair) with 3 stations (VLBA-KP, VLBA-LA, and OVRO 40-m). In August 1990 we obtained 14 hours of data (4 sections 
per source pair) with 5 stations (VLBA-PT, VLBA-LA, VLBA-FD, VLA (1 antenna), and OVRO 40-m). We will shortly (November 1990) be extending our tests to global baselines at $3.6 \mathrm{~cm}$, using a similar strategy as before. A comparison of similar data at 3.6 and $6 \mathrm{~cm}$ should allow us to assess the relative importance of tropospheric and ionospheric phase fluctuations in limiting the phase referencing technique.

\section{DATA REDUCTION}

The Block-II correlator in Pasadena uses a relatively simple model for fringetracking. Subsequent processing is done in AIPS. After careful editing, the task CALIB is used to obtain global fringe-fitting solutions for the delay, phase-rate, and phase in each ( $\leq 1.5$ minute) interval on the reference source. With the task CLCAL we interpolate the reference delays and phases (using the phase and phase-rate solutions) to the times when the targets were observed. These values are then subtracted from the target source data using the task SPLIT.

We are developing software to remove the phases introduced by the correlator model, so restoring total phases. We can then use geodetic software available at JPL (MASTERFIT or SPRINT), and incorporate tropospheric/ionospheric modeling with weather/WVR input. Thus, as pointed out by Lestrade et al. (1990, see also these proceedings), the final results need not be limited by the accuracy of the correlator model. The plots shown in this progress report still contain residual phase slopes (with a diurnal sinusoidal signature) due to inaccuracies of up to $10 \mathrm{~m}$ in some VLBA antenna coordinates used during correlation.

\section{PRELIMINARY RESULTS}

The 6-cm data obtained and reduced thus far are most relevant to analyse the phase variability on $\leq 1000 \mathrm{~km}$ baselines over timescales between a few minutes and an hour. We have insufficient data to differentiate the results for different baseline lengths; those shown are typical.

Figure 1 shows typical cases in which an interpolation using only brief phase-calibrator scans at the start and end of a $(\sim 45$ minute) section helps to improve the phase stability on the target source. Such a procedure removes phase slopes due to clock drifts. The use of all phase-calibrator data, at 3 minute intervals, leads to very significant additional improvements, presumably because we now calibrate out atmospheric phase effects.

As our figures show, after correction at 3 minute intervals the residual phase variations are 0.5-1.0 radian peak-to-peak. From Figure 2 we note that the results are often comparable at target-reference separations ranging from $0.4^{\circ}$ to $3.7^{\circ}$. This agrees with the predictions of Treuhaft and Lanyi (1987) about the phase variations caused by the wet part of the troposphere. However, there is a period in our November 1989 dataset for which the phase stability is poorer at the larger separations. Evidently, spatial variations over several degrees sometimes do dominate over temporal variations in a few minutes. It is unclear whether this is due to the wet or the dry troposphere, or even to the ionosphere. We hope to accumulate more statistics to determine how spatial variations relate to weather conditions and other parameters. 
A more elaborate geometric/atmospheric model may allow additional improvements of the achieved phase stability. Furthermore, the interpolation scheme used in phase referencing should be matched to the temporal structure function of the phase variations. Some of our data may be affected by $\sim 0.5$ radian peak-to-peak atmospheric phase variations on a timescale of a few minutes. In that case direct linear interpolation of the reference phases is superior to interpolation using both phases and phase-rates.

From our preliminary analysis we conclude that phase-referencing allows the coherence time to be significantly extended. There are good prospects for making limited dynamic range images of weak sources with baselines out to at least $1000 \mathrm{~km}$, using a calibrator at a separation as large as several degrees.

This work was supported by the National Science Foundation, grant number AST-8814554. We have used the VLBA of the National Radio Astronomy Observatory, operated by Associated Universities, Inc., under Cooperative Agreement with the National Science Foundation.
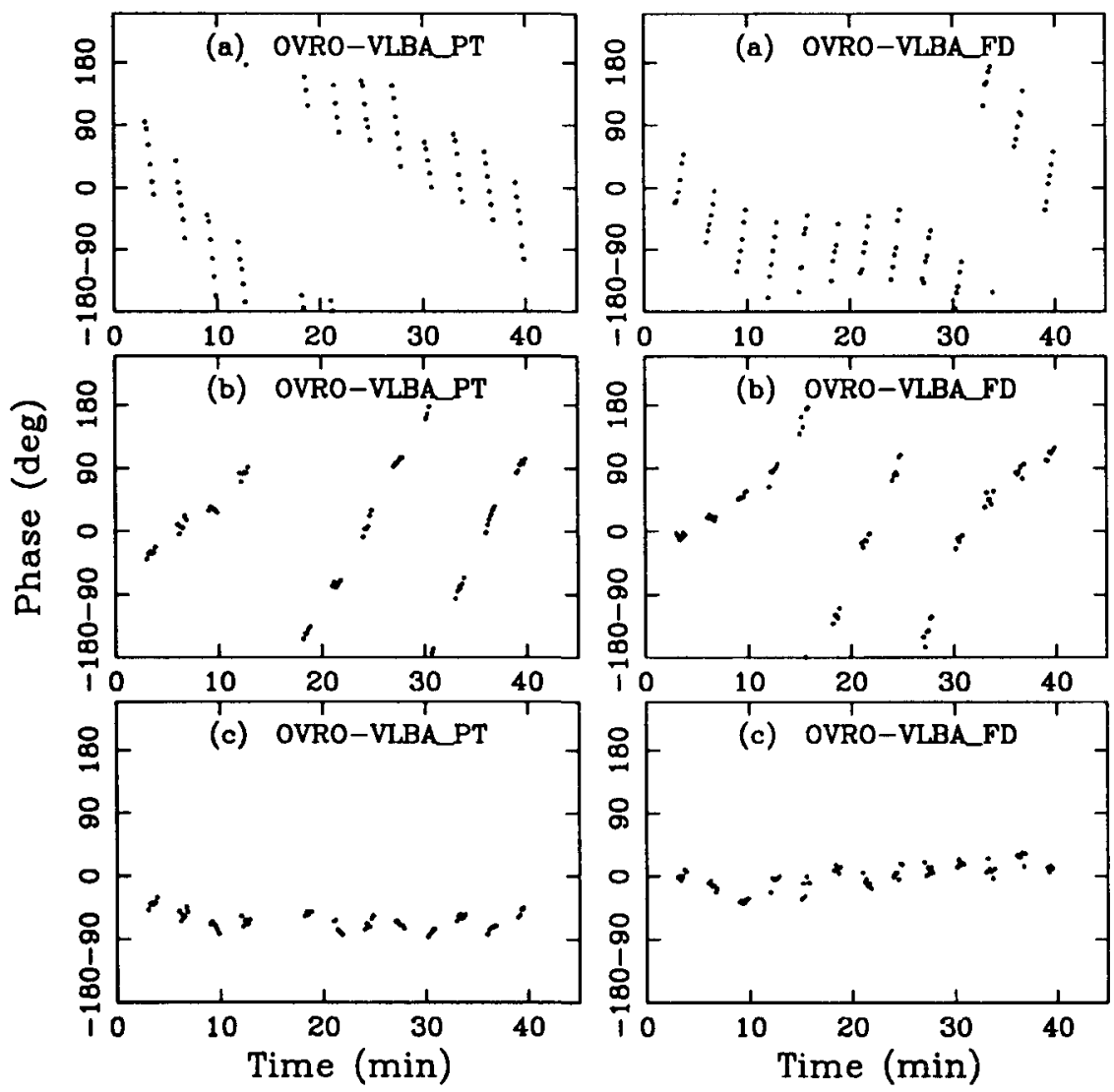

Fig. 1. Illustration of the phase stability achieved using phase referencing for $1633+398$ (2.2 $2^{\circ}$ from 3C 345). (a) Raw Phases. (b) Phases calibrated with a reference scan at the start and end of the 40-minute section only. (c) Phases calibrated with a reference scan every 3 minutes. 

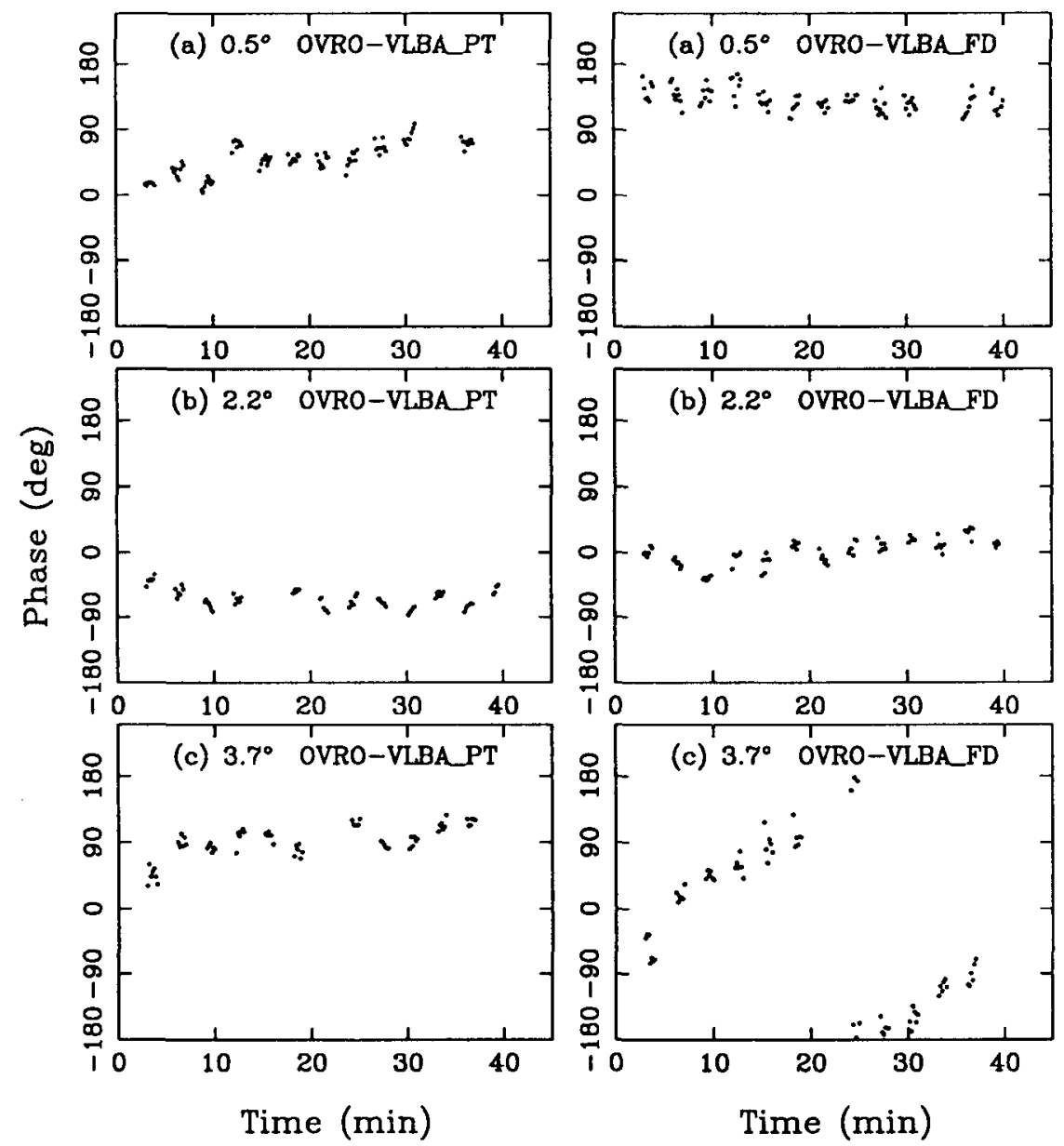

Fig. 2. Illustration of the phase stability achieved as a function of the reference-target separation (using reference scans every 3 minutes). (a) $0.4^{\circ}$ separation $(1638+398)$. (b) $2.2^{\circ}$ separation $(1633+382)$. (c) $3.7^{\circ}$ separation $(1624+416)$.

\section{REFERENCES}

Alef, W. 1988, in IAU Symposium 129, eds M.J. Reid and J.M. Moran, p. 523. Lestrade, J.-F., Rogers, A.E.E., Whitney, A.R., Niell, A.E., Phillips, R.B. 1990, Astron. J. 99, 1663.

Lestrade, J.-F. 1991, These Proceedings, "VLBI phase referencing: astrometry and imaging".

Patnaik, A.R., Brown, I.W.A., Wilkinson, P.N., and Wrobel, J. 1991, These Proceedings, "Phase calibrators for MERLIN".

Treuhaft, R.N., and Lanyi, G.E. 1987, Radio Science 22, 251. 